\title{
INVESTIGACIONES
}

\section{Affordance pedagógico del cine en la enseñanza de la reflexión epistemológica}

\author{
Pedagogical affordance of cinema in the teaching of epistemological reflection
}

\author{
Tomas Fonatinez-Ruiz, Nora Casimiro Urcos ${ }^{b}$, \\ Rosa Gonzáles Llontop ${ }^{c}$, Italo Jiménez Idrovo ${ }^{d}$ \\ a Universidad Técnica de Machala, Ecuador. Correo electrónico: tfontaines@ hotmail.com

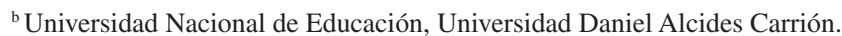 \\ c Universidad Nacional Pedro Ruiz Gallo, UNPRG. \\ d Universidad Técnica de Machala, Ecuador.
}

\section{RESUMEN}

El estudio devela el affordance pedagógico del cine en el aprendizaje de la reflexión epistemológica. Los datos discursivos se analizaron mediante el uso del método de clustering no supervisado bisecting K-means, cuya interpretación se realizó a partir de los lemas en sus contextos significantes. Se concluye que el uso pedagógico del cine permite: 1) una mayor comprensión de conceptos epistemológicos y metodológicos; 2) involucran al formando con la autocorrección de dudas desde la cotidianidad de su percepción, 3) impulsan la construcción de conceptualizaciones significantes; 4) personalizan las abstracciones que subyacen al pensamiento epistemológico, 5) promueven la construcción de dispositivos lingüísticos que sirven de argumentos en las decisiones vinculadas con el desarrollo de la investigación.

Palabras clave: enseñanza de la epistemología, usos, formación de investigadores, métodos de investigación

\begin{abstract}
The study reveals the pedagogical affordance of cinema in the learning of epistemological reflection. The discursive data were analyzed by using the non-supervised clustering method bisecting k-means, which interpretation proceeded to the location of the phrases on their significant contexts. It is concluded that the pedagogical use of the cinema allows: 1) a better understanding of epistemological and methodological concepts; 2) involving the learner with the self-correction of doubts from his/her everyday perception, 3) boosting the construction of significant conceptualizations; 4) personalizing abstractions underlying epistemological thinking; 5) promoting the construction of linguistic devices that serve as arguments in decisions related to the development of research.
\end{abstract}

Key words: epistemology teaching, utilization, researchers training, research methods 


\section{INTRODUCCIÓN}

En la actualidad universitaria hacer investigación se constituye en la posibilidad de existir. Investigar, lejos de ser un requisito para la obtención de la titulación o un determinado ascenso, es un estilo de vida. La dinámica social de los centros universitarios gira en torno a la promoción y posicionamiento de líneas, grupos y proyectos de investigación como condición para incrementar el estatus individual y colectivo del centro de formación donde ésta se desarrolla. A tal nivel llega la situación, que los intercambios interinstitucionales se hacen atractivos cuando la institución a la que se aspira llegar, presenta una estructura de investigación madura y consolidada, caracterizada por la exigencia de publicaciones en revistas científicas de alto impacto y con posición intercuartílica privilegiada, como es ya cotidiano en universidades europeas norteamericanas y de manera creciente, en Latinoamérica.

En este devenir los noveles docentes-investigadores universitarios, con el afán de ponerse a tono con tal exigencia, asumen de manera voluntaria, circunstancial o impuesta una determinada orientación temática, epistémica y metodológica para llegar a conocer el objeto de su interés, situación que les resulta compleja y liberadora a la vez. La dificultad de la tarea radica en la comprensión de lo abstracto del andamiaje discursivo y teórico empleado en la construcción de la realidad y su transferencia a la acción cotidiana. Lo liberador está en argumentar frente a cualquier comunidad científico-profesional, las decisiones metodológicas tomadas al momento de investigar. Este hecho reclama el desarrollo de estrategias formativas que permitan el aprendizaje de tales saberes con significación, transferencia y alejado de las pasiones humanas que deforman la imagen de la ciencia (Fernández, Gil , Carracosa, Cachapuz, y Praia, 2002; Paz Penagos, 2008). Con base en lo dicho, el presente estudio pretende develar el affordance pedagógico del cine en el aprendizaje de la reflexión epistemológica en docentes de la Universidad Técnica de Machala, Ecuador, sometidos a un proceso de formación de competencias investigadoras aplicables a sus áreas disciplinares.

Esta investigación ofrece un modo distinto de entender la valoración del cine como opción pedagógica, al apreciar los significados y usos potenciales del mismo desde una perspectiva discursiva colaborativa y cualicuantitativa. Los significados que se esconden en el discurso son develados mediante clúster formados a partir de lematizaciones. Los datos objeto de análisis testimonian cómo el novel investigador usa los recursos del cine para darle forma a lo abstracto que resultan las prescripciones epistemológicas y metodológicas en el hecho investigador, lo cual resulta imprescindible para afrontar lo esnobista, prescriptivo, dogmático, anárquico o liberador que pueda resultar su vinculación con el método al momento de investigar.

En este estudio también se promueven escenarios pedagógicos para movilizar la reflexión en torno al dogmatismo versus libertad metodológica como consecuencia de la reflexión epistemológica. Se espera que el novel docente investigador enrole una postura crítica y singular al responder interrogantes del tipo: ¿existe un modo de concebir la producción del conocimiento?, o ¿es posible generar adecuaciones metodológicas para atender las características estructurales y funcionales del objeto de estudio?. Estos espacios didácticos reclaman su inaplazable atención cuando de formar investigadores se trata y ello se debe a que: a) El conocimiento es un mecanismo de acercamiento a un objeto, que reclama un enfoque para hacer inteligible la realidad, no es la realidad en- 
sí (Nicolás y Frápoli, 1997), b) La ciencia procura aprehender la realidad y se provee de ciertas herramientas teórico-epistemológicas para reflexionar sobre la legitimidad del conocimiento al que llega (Guerrero, 2009), c) Por extensión, tendemos a pensar que el conocimiento es la realidad; entonces, lo que hace la epistemología es proveernos de recursos para comprender cómo nos acercamos a esa realidad, mediante el análisis de las causas, origen, y condiciones en las que se produce el conocimiento. d) La investigación es un recurso para aprehender la realidad y la epistemología vendría a ser su eje central. Lamentablemente la ciencia ha buscado separarse de ese carácter inicial, fundamentada en sus logros y en el predominio de la técnica, por lo que la epistemología es reemplazada por una construcción metodológica útil para los intereses de quienes dominan los paradigmas del conocimiento (Kuhn, 2004).

\section{GIBSON Y SU TEORÍA DEL AFFORDANCE}

El corolario central de la llamada Teoría del affordance o de los usos propone que los objetos sugieren usos probables, por lo tanto, no existe un sentido único de aplicabilidad de un elemento. El contexto, la naturaleza del objeto y la predisposición del sujeto serán los elementos condicionantes de la aparición de un affordance. Las investigaciones indican que este concepto lo difunde Gibson en el año 1979 en la psicología ecológica, demostrando que la interacción del sujeto con el objeto en el ambiente, va a determinar los usos probables que este último tenga (Costall, 1995; Gibson, 2000; Turvey, 1992; Hutchby, 2001; Borghi \& Riggio, 2009; Da silva Oliveira y Tosi Rodigues, 2006; Baerentsen \& Trettvik, 2002).

Entre las propiedades del affordance destacan las siguientes: a) son oportunidades para actuar. La estructura del objeto es percibida como una posibilidad para resolver situaciones deficitarias en el sujeto; b) los affordances son una intersección entre las propiedades físicas de los objetos y sus posibilidades de uso; c) a pesar de ser interdependientes al sujeto, es éste quien de acuerdo con sus capacidades valora las condiciones del objeto; d) el affordance siempre va a estar presente en el objeto aunque el sujeto decida o no utilizarla; e) el objeto siempre estará en posesión de sus condiciones estructurales, las cuales no son creadas como respuesta contingente ante las demandas del sujeto.

Este sencillo pero complejo concepto encierra la posibilidad de aumentar los usos potenciales que tengan los objetos en contexto. Mas allá del uso intencional al que estamos acostumbrados, existen múltiples esquemas de interacción que aún no se perciben y que potencialmente pueden dar respuestas a requerimientos que aún reclaman atención. De modo particular, en el caso que nos ocupa, mirar el cine desde la visión del affordance, sugiere que en el marco de las libertades epistemológicas asumidas, empezarán a develarse modos de interacción entre el conocimiento, la ciencia y los procesos de aprendizaje.

\section{POSICIONES EPISTEMOLÓGICAS: ¿CUÁNTAS SON?, ¿CUÁNDO SURGEN?}

La Epistemología nos remite a la pregunta ¿En qué condiciones se da el conocimiento con el que interpretamos los fenómenos que configuran la realidad? La respuesta a esta interrogante, ha sido causal de debates en la historia de la ciencia (Altisen, 2001; Bunge, 2002), donde se perciben convergencias y divergencias conceptuales y operativas respecto 
a la identificación de argumentos consistentes sobre la construcción de la investigación. La contraposición obedece a la divergencias existentes al momento de definir las categorías ontológicas, gnoseológicas y metodológicas que intevienen en la invención, construcción o descubrimiento del conocimiento. A tal efecto, a continuación se plantea una síntesis de cinco posiciones epistémicas que más se atienden en la literatura epistémico-metodológica, a saber: escepticismo, empirismo, racionalismo, idealismo, realismo, tomando como referencia la información descrita en la cuadro 1.

Cuadro 1. Análisis categórico de posturas epistemológicas

\begin{tabular}{|c|c|c|c|c|c|}
\hline \multirow{2}{*}{$\begin{array}{l}\text { Categorías de } \\
\text { contraste }\end{array}$} & \multicolumn{5}{|c|}{ Posiciones epistemológicas } \\
\hline & Escepticismo & Empirismo & Racionalismo & Idealismo & Realismo \\
\hline $\begin{array}{l}\text { Quién es el } \\
\text { hombre }\end{array}$ & $\begin{array}{l}\text { Sujeto que } \\
\text { conoce }\end{array}$ & $\begin{array}{l}\text { Sujeto } \\
\text { provisto de } \\
\text { sentidos }\end{array}$ & Cosa pensante & $\begin{array}{l}\text { Materialización } \\
\text { de la Idea de } \\
\text { hombre }\end{array}$ & $\begin{array}{l}\text { Ser humano } \\
\text { (concreto) }\end{array}$ \\
\hline $\begin{array}{l}\text { Qué es la } \\
\text { realidad }\end{array}$ & $\begin{array}{l}\text { Objeto del cual } \\
\text { no se puede } \\
\text { tener certeza }\end{array}$ & $\begin{array}{l}\text { Aquellas } \\
\text { impresiones } \\
\text { que obtenemos } \\
\text { por los } \\
\text { sentidos }\end{array}$ & $\begin{array}{l}\text { Cosa pensante } \\
\text { (hombre) Cosa } \\
\text { extensa } \\
\text { (mundo) cosa } \\
\text { infinita (dios) }\end{array}$ & $\begin{array}{l}\text { La idea (en su } \\
\text { representación } \\
\text { espacio- } \\
\text { temporal) }\end{array}$ & La cosa \\
\hline $\begin{array}{l}\text { Quién es el } \\
\text { objeto del } \\
\text { conocimiento }\end{array}$ & $\begin{array}{l}\text { (Por deducción) } \\
\text { Aquello a lo que } \\
\text { intentan } \\
\text { aprehender los } \\
\text { juicios }\end{array}$ & $\begin{array}{l}\text { La fuente de } \\
\text { las } \\
\text { impresiones } \\
\text { que afectan los } \\
\text { sentidos }\end{array}$ & $\begin{array}{l}\text { Aquello que } \\
\text { puede ser } \\
\text { pensado }\end{array}$ & $\begin{array}{l}\text { La sombra de la } \\
\text { idea (lo sensible) }\end{array}$ & La cosa \\
\hline $\begin{array}{l}\text { Quién es el } \\
\text { sujeto del } \\
\text { conocimiento }\end{array}$ & $\begin{array}{l}\text { Ser capaz de } \\
\text { cuestionamiento }\end{array}$ & $\begin{array}{l}\text { Ente capaz de } \\
\text { recibir } \\
\text { impresiones } \\
\text { por medio de } \\
\text { los sentidos }\end{array}$ & Cosa pensante & $\begin{array}{l}\text { Ente capaz de } \\
\text { retrotraerse a su } \\
\text { estado ideal } \\
\text { (anamnesis) } \\
\text { original, generar } \\
\text { categorías o } \\
\text { captar y dejarse } \\
\text { captar por el } \\
\text { Espíritu }\end{array}$ & $\begin{array}{l}\text { Ser humano } \\
\text { (concreto) }\end{array}$ \\
\hline $\begin{array}{l}\text { Concepción } \\
\text { del dato/ } \\
\text { Evidencia }\end{array}$ & $\begin{array}{l}\text { Presentación } \\
\text { teórica temporal } \\
\text { (Hay dato } \\
\text { provisional, } \\
\text { pero no } \\
\text { evidencia) }\end{array}$ & $\begin{array}{l}\text { El resultado } \\
\text { que arrojan las } \\
\text { impresiones } \\
\text { sensibles }\end{array}$ & $\begin{array}{l}\text { Aquello que } \\
\text { puede ser } \\
\text { pensado y se } \\
\text { presenta de } \\
\text { manera clara y } \\
\text { distinta }\end{array}$ & $\begin{array}{l}\text { Una construcción } \\
\text { provisional para } \\
\text { apuntalar la } \\
\text { evidencia de la } \\
\text { idea a la que ese } \\
\text { dato refiere }\end{array}$ & $\begin{array}{l}\text { Información } \\
\text { empírica } \\
\text { verificable }\end{array}$ \\
\hline $\begin{array}{l}\text { Concepción } \\
\text { del error }\end{array}$ & $\begin{array}{l}\text { Aquello de lo } \\
\text { cual no se tiene } \\
\text { certeza }\end{array}$ & $\begin{array}{l}\text { La idea } \\
\text { desprovista de } \\
\text { un referente } \\
\text { empírico } \\
\text { (sensible) }\end{array}$ & $\begin{array}{l}\text { La idea } \\
\text { formada a } \\
\text { partir de } \\
\text { simples } \\
\text { impresiones } \\
\text { sensibles }\end{array}$ & $\begin{array}{l}\text { Pensar que lo } \\
\text { sensible es lo } \\
\text { real }\end{array}$ & $\begin{array}{l}\text { No hay error, } \\
\text { las cosas } \\
\text { existen. }\end{array}$ \\
\hline
\end{tabular}




\begin{tabular}{|c|c|c|c|c|c|}
\hline $\begin{array}{l}\text { Proceso } \\
\text { metódico }\end{array}$ & $\begin{array}{l}\text { Negación de } \\
\text { posibilidad de } \\
\text { verdad } \Rightarrow \\
\text { Abstención del } \\
\text { juicio } \Rightarrow \text { idea/ } \\
\text { conocimiento }\end{array}$ & $\begin{array}{l}\text { Percepción } \\
\text { por la } \\
\text { experiencia } \\
\text { (sentidos) } \Rightarrow \\
\text { Asociación } \\
\text { mental } \Rightarrow \\
\text { idea/ } \\
\text { conocimiento }\end{array}$ & $\begin{array}{l}\text { Percepción } \\
\text { por la razón } \\
\text { (intelecto) } \Rightarrow \\
\text { Deducción } \Rightarrow \\
\text { idea/ } \\
\text { conocimiento }\end{array}$ & $\begin{array}{l}\text { Presupuesto (las } \\
\text { ideas existen por } \\
\text { sí mismas) } \Rightarrow \\
\text { Percepción } \\
\text { intelectiva de las } \\
\text { ideas } \\
\text { (aprehensión) } \Rightarrow \\
\text { idea/ } \\
\text { conocimiento }\end{array}$ & $\begin{array}{l}\text { Presupuesto (la } \\
\text { realidad existe } \\
\text { por sí misma) } \\
\Rightarrow \text { Adecuación } \\
\text { del intelecto a } \\
\text { la realidad } \Rightarrow \\
\text { Realidad/ } \\
\text { conocimiento }\end{array}$ \\
\hline Representante & Pirron de Elis & D. Hume & R. Descartes & Platón & Aristóteles \\
\hline
\end{tabular}

\subsection{ESCEPTICISMO}

El escepticismo surge a mediados del siglo IV a.C., como una posición epistemológica que al no poder tener un referente último de la verdad, procura no emitir juicios últimos sobre la misma. Contrariamente a ser una posición heurística, hermenéutica o teórica, nos evita asumir cualquier cosa como verdadera, sin tener los elementos necesarios para considerarla como tal (Nussbaum, 2003). Nace como una búsqueda de conocimiento diferente a las ciencias formales, inicialmente opuesta al predominio de una vía de comprensión de la realidad (Olmedo, 1992).

\subsection{EMPIRISMO}

La posición empirista considera que la única información válida para tener conocimiento verdadero es aquella que nos proveen los sentidos y que luego se imprime (Locke, 1985) en nuestras mentes, pudiendo ser entendida como una impresión de sensación o de reflexión (Hume, 1984). Las primeras pueden ser innatas, mientras que las segundas parten de alguna impresión original. La escuela empirista se ubica en el siglo XVII con autores con Hume, Locke, Berkeley.

\subsection{RACIONALISMO}

La información que se puede considerar como verdadera, no es aquella que nos proveen los sentidos, puesto que éstos nos engañan (Descartes, 2009), mientras que el conocimiento adquirido por vía intelectiva no ofrece sombra de duda. Así, nuestra primera fuente de conocimiento es la razón, entendida como la facultad mental de generar y aprehender ideas. La escuela racionalista surge en Europa a finales del siglo XVI con autores como Descartes, Spinoza, Leibniz; es el referente directo para la Ilustración del siglo XVIII puesto que comienza a crear un clima y un modo distinto de construcción del conocimiento, diferente al de la Edad Media.

\subsection{IDEALISMO}

Los presocráticos procuraron responder a la pregunta por el origen del cosmos, de todo lo existente; sin embargo, los filósofos clásicos sistematizan estos conocimientos y asumen 
otro enfoque que se fundamenta en la idea de que la realidad no está limitada a lo realexistente, sino que el único conocimiento verdadero es aquel que se encuentra en las ideas, esto es a lo que Platón llama "episteme" o conocimiento verdadero (Platón, 1992; Altisen, 2001) que debe su realidad a la percepción del sujeto y no a la existencia del objeto mismo. Esta postura ideológica puede rastrearse desde el siglo V a. C., hasta comienzos del siglo XIX, distinguiéndose incluso tres grandes corrientes: Idealismo clásico (Platón), Idealismo trascendental (Kant) e Idealismo Absoluto (Hegel).

\subsection{REALISMO}

El realismo considera que los objetos tienen existencia por sí mismos, independientemente de ser percibidos o no, lo que en el plano de la epistemología equivale a entender la relación entre fenómeno y constructo, donde el primero se refiere a lo que se muestra en la realidad; mientras que el segundo, a la construcción teórica que puede adquirir carácter de existente y para los idealistas de real (Bunge, 2002). Es decir, el fundamento de aquello que se conoce sobre un objeto es el objeto mismo y no su verdad (De Vries, 1945). En términos generales, esta postura puede ser percibida desde los presocráticos en el siglo VII a. C., hasta la actualidad donde la Ciencia o la Filosofía de la ciencia conservan sus presupuestos fundamentales.

\section{EL FUNCIONAMIENTO PRESCRIPTIVO DE LA EPISTEMOLOGÍA FRENTE A LA METODOLOGÍA}

Las posturas epistemológicas condicionan las secuencias operativas de investigación que se aplican durante la concepción de un proceso de investigación y sus formas operativas. A continuación se muestran algunas evidencias de ello: 1) El novum organum baconiano es muestra de la inquietud de su autor por dominar la naturaleza y generar un modo de interpretación sistemática para todas las ciencias (Dear, 2001). El conocimiento se entiende como una representación exacta de la realidad, alcanzada mediante la inducción (Bacon, 2011). La representación de ello se observa en la Nueva Atlántida (Bacon, 2006), donde se exponen los roles del sujeto y los procesos metódicos de investigación, que pasan de la observación a la experimentación, de allí a la creación de axiomas y con base en éstos a la producción de alternativas de aprehensión de las formas más generales de la naturaleza. 2) Descartes (2009) y su obsesión por justificar el uso del método como condición para la obtención del conocimiento. 3) El Círculo de Viena y la unificación de la ciencia, mediante el siguiente núcleo temático: i) la verificabilidad es el criterio de demarcación que legitima lo que es o no científico; ii) la comunicación científica, se realiza en lenguaje fisicalista, ya que la física, las matemáticas y la lógica son las ciencias a imitar; iii) la inducción probabilística permite la generalización de teorías mediante la inferencia estadística; iv) se requiere de un método único para la institución científica Quesada Sánchez, 2004; Álvarez Ortega, 1999).

Tanto Bacon como Descartes y los vieneses se esforzaron en crear una estructura lógicometodológica que explicase modos únicos de acercarse al conocimiento científico. En todos se observan argumentos que ponen el método por encima de cualquier circunstancia. Asumen actitudes dogmáticas, cuya práctica y error es que no justifican lo afirmado, 
cerrándose a modos divergentes de pensar (Orden Jiménez, 1998). Terminan haciendo del método un axioma y aquello que nace siendo un medio, se constituye en un fin en sí mismo. Lo que inicialmente es un argumento, pasa a ser una disposición normativa que fronteriza lo que es o no científico.

$\mathrm{Al}$ aceptar esta visión, el novel docente-investigador se hace un reproductor acrítico de los dogmas metodológicos frente a la ciencia (Ramírez Sánchez, 2002), poniendo en evidencia la voluntad de poder (Nietzsche, 2000; Izquierdo, 2000) de quienes conforman los nichos académicos. Aún en los centros universitarios las actitudes impositivas siguen existiendo y en la pretensión de mayor cientificidad y rigor se terminan creando esquemas únicos para cosas múltiples. Se sigue avivando la adopción de la ciencia como producto desligado de su carácter histórico y contextual, tal como se expresa en la distinción del contexto de justificación de Reichenbach (Estany, 2006; Chá Larrieu, 2002). Seguimos "reduciendo la ciencia al conocimiento científico" (Echeverría , 1998).

Al ser una actividad, la ciencia tiene una dimensión diacrónica; en su construcción intervienen la historia, la sociología, la psicología, entre otras. En la interacción está la génesis del conocimiento, por tanto la mirada sobre la producción y vigencia de las teorías científicas deben integrar además de lo cognitivo, las dimensiones sociales, y psicoafectivas propias de la naturaleza humana. Justamente en este argumento encontramos el sentido de negación sobre la existencia de "un modo único" de producir conocimiento.

Esta ha sido una disquisición que desde los años sesenta se realiza con intensidad como una respuesta a la actitud dogmática ya descrita. Los trabajos de Kuhn (1989) dejan establecido que la ciencia está condicionada por la trama sociohistórica del momento. La ciencia es testimonio de su tiempo, en consecuencia, se ve sometida a constantes cambios y tensiones. También Lakatos $(1976 ; 1999)$ en sus programas de investigación genera mecanismos de acomodos progresivos para responder a las demandas externas. Si bien su núcleo fuerte es incorruptible, los cinturones protectores y sus heurísticas son garantes de la adaptabilidad. La ciencia se gesta en contextos, por tanto, no pueden haber conjeturas ingenuas ni falsaciones totales.

Por su parte Laudan (1978) indica que los cambios en la percepción de la teoría y el método en general, más allá de ser físicos, son conceptuales; es decir, la idea de que las teorías coexistan es más una norma que una utopía. En consecuencia, la valoración teórica responde a la percepción local más que a un criterio axiomático. Para cerrar este flujo argumentativo, Feyerabend $(1987 ; 2008)$ nos invita a pensar en que no existe un método, existen los métodos. Se niega a aceptar la neutralidad de la ciencia. Propone que el método más que un fin es un medio reinventado de cara a los requerimientos de la investigación en desarrollo.

Como se puede observar, habrán tantas adecuaciones epistémicas y metódicas como objetos y tiempos históricos existan (Castro Nogueira, Castro Nogueira, y Morales Navarro, 2008; Biber \& Nagy, 2010) de allí la idea de enseñar a investigar con base en la reflexión metacientífica, a fin de: a) confrontar posiciones intelectuales que sacralizan el método por encima del objeto, b) construir perspectivas personales sobre la práctica que se estudia (Acevedo-Díaz, Vazquez-Alonso, Manassero-Mas, y Acevedo-Romero, 2007), c) romper con las deformidades sobre la ciencia que se han venido socializando y que logran tener un estatus de verdad, cuando en resumidas cuentas solo son perspectivas individuales que se consumen y transmiten a los miembros de determinadas comunidades discursivas (Fernández et al., 2002). 


\section{METODOLOGIA DEL ESTUDIO}

La rutina metodológica empleada corresponde con los lineamientos de la investigación no experimental, transeccional descriptiva, con un nivel de profundidad descriptivo (Padrón, 2007). Se contó con la participación de 102 profesores de la Universidad Técnica de Machala, Ecuador, distribuidos de la siguiente manera: $20 \%$ adscritos a la Unidad Académica de Ciencias Químicas y de la Salud, $45 \%$ a Ciencias Sociales y 35\% a Ciencias Empresariales. El desarrollo del estudio siguió las fases que se nombran a continuación:

Fase 1. Encendido de las alertas: en este primer estadio de la intervención, los docentes identificaron actitudes dogmáticas alrededor de las esferas epistemológicas y metodológicas de la investigación mediante el análisis crítico del micro film "El mito de la caverna de Platón" y del film titulado Galileo, dirigido por Losey (1974). Durante dos jornadas de trabajo de 5 horas cada una, se realizaron las siguientes acciones: a) introdución al tema por parte del facilitador; b) presentación del micro film de Platón; c) discusión socializada para develar la dimensión antropológica, ontológica y epistemológica que subyace al mito y cómo estos elementos se personalizan en la actitud del profesorado ante la investigación (resistencias y aperturas); d) presentación del film de Galileo; e) discusión socializada y construcción colectiva de aprendizajes respecto a: apertura de los tiempos históricos a los avances del conocimiento humano. Coexistencias de concepciones epistemológicas. Diseños metodológicos emergentes como signo de inventiva y creatividad del investigador para aprehender el objeto y a propósito de ello, describirlo, explicarlo y aplicar situaciones de mejora en favor de la calidad de vida del colectivo. Construcción de la verdad como artefacto social. A modo de síntesis, la intención de esta fase fue que los participantes tomaran conciencia de su actitud hacia el conocimiento y, al mismo tiempo, mostrar cómo los tiempos históricos viabilizan u obstaculizan el crecimiento de la actividad científica.

Fase 2. Identificación de la relación condicional entre enfoques epistemológicos y sus secuencias operativas de investigación: la intención de esta fase fue analizar los componentes diacrónicos y sincrónicos de los enfoques epistemológicos y los procesos de investigación (Padrón, 2007; Camacho, 2000). Se llevó a cabo durante tres sesiones de 5 horas cada una. En la primera sesión se trabajaron discusiones socializadas respecto a los componentes estructurales y dinámicos de los enfoques epistemológicos que previamente identificamos en el apartado tres del presente documento. Al cierre de esta jornada se produjo la construcción de un cuadro comparativo análogo al observado en la tabla 1. La segunda sesión consistió en visualizar las categorías epistemológicas y metodológicas en los personajes y trama del film: "El aceite de la vida" (Miller y Miller, 1992). Al final del proceso se produjo una discusion socializada alrededor de las categorías observadas según lo indicado en la tabla 1. La tercera sesión de trabajo fue análoga a la segunda, con la diferencia que se empleó el film “Gorilas en la niebla” (Clegg y Glimcher, 1988).

Fase 3. Valoración de la experiencia y construcción del affordance: Esta fase consistió en ubicar la información valorativa de los participantes sobre los usos del cine. Para ello, se envió por correo electrónico un formulario de preguntas abiertas, que los participantes debían contestar de acuerdo con sus impresiones. Las preguntas eran las siguientes: 1) De qué modo el cine contribuyó con la comprensión de los saberes 
epistemológicos vinculados con la investigación; 2) De qué modo el uso del cine fomentó cambios conceptuales respecto al aprendizaje de saberes epistemológicos y su vinculación con la investigación. Para llevar a cabo el análisis del corpus obtenido de las respuestas de los participantes se realizó un análisis de clúster mediante el uso del método de clustering no supervisado bisecting K-means (Marín y Branch, 2008), calculado con el software T-lab, version 8. Esta técnica devela los núcleos semánticos de la expresión discursiva, tal como se observan en las investigaciones de Caputo, (2013); Barello, Leone, Danese, \& Vegni, (2014).

\section{PRESENTACIÓN Y ANÁLISIS DE LOS RESULTADOS DEL ESTUDIO}

Los resultados de la investigación se estructuran en dos momentos que son: a) caracterización del corpus objeto de análisis; b) proceso de reducción semántica e interpretación de los resultados. El desarrollo de estos apartados se muestra a continuación:

Caracterización del corpus: el corpus en estudio se deriva de las respuestas de los docentes a un cuestionario ad hoc cuyo objetivo fue valorar el affordance pedagógico del cine en la enseñanza de la epistemología. Esta evaluación se hace en el marco del desarrollo de un programa de formación de competencias investigadoras en los investigadores nóveles de la Universidad Ténica de Machala, Provincia de El Oro, Ecuador, cuya finalidad, en este apartado, fue promover el uso consciente del método en congruencia con la naturaleza del objeto de estudio, el perfil del investigador, su área de conocimiento y lógicamente el objetivo del estudio. Este corpus se tipifica como de referencia, y está constituido por 2637 palabras y 16455 repeticiones. Al ser un corpus empleado para analizar la semántica de los usos, el número de palabras muestradas se consideran válidas según indican Torruella y Llisterri, (1999).

Proceso de reducción semántica e interpretación de los resultados: la identificación de los clúster se diferenció en virtud de cada una de las preguntas realizadas. A continuación se muestran:

\section{Pregunta 1. De qué modo el cine contribuyó con la comprensión de los saberes epistemológicos vinculados con la investigación.}

Como se puede observar en la figura 1, se han formado dos factores que explican el $75,27 \%$ de la varianza asociada a la posición de los profesores respecto al modo en que el uso del cine contribuye a la comprensión de los saberes epistemológicos. Este porcentaje representa el caudal de energía textual (Torres-Moreno, Molina, y Sierra, 2010) identificada en el discurso y al mismo tiempo revela la homogeneidad de opiniones de los hablantes. De esta manera, si se considera que el discurso es un medio de construcción de realidades (Carrillo Guerrero, 2006), tendría sentido asumir que los cuatro clústers formados (ver cuadro 2) testimonian, que mediante el cine se logra un mayor y mejor aprendizaje de los saberes epistemológicos y se comprende el modo en que éstos se vinculan con la investigación.

La interpretación de los clúster se realiza mediante la ubicación del lema en el contexto discursivo. El primero de ellos muestra el potencial del cine como medio para la 
obtención de los aprendizajes. Se reconoce que la observación de hechos reales favorece el cruce de ideas para la construcción de aprendizajes vinculados con la epistemología y la investigación. El cine es una ventana para ilustrar cruces de ideas en torno a la ciencia e investigación, al tiempo que permite lograr un aprendizaje con menor esfuerzo y mayor rendimiento.

El segundo clúster revela que mediante el cine se produce interacción entre la información y la duda del novel docente-investigador. Mediante el cine se realizan abstracciones y se observan diversos criterios frente al conocimiento, indicando que la investigación se puede realizar desde cualquier punto de referencia o conocimiento previo.

El tercer clúster muestra la adaptabilidad de los procesos metodológicos a los objetos de investigación, de allí la existencia de diversos métodos. El cine contribuye a mostrar variados modos de investigar y la necesidad de no ahogar la investigación para favorecer ideas preconcebidas.

Finalmente el cuarto clúster revela una inclinación didáctica en el uso del cine. Se reconoce que el desmontaje de los vídeos muestran la vinculación de conceptos epistemológicos con la práctica de la investigación y el modo en que se perciben desde el ejercicio práctico del estudio. Hay conciencia de la relación condicional entre enfoques epistemológicos e investigación científica.

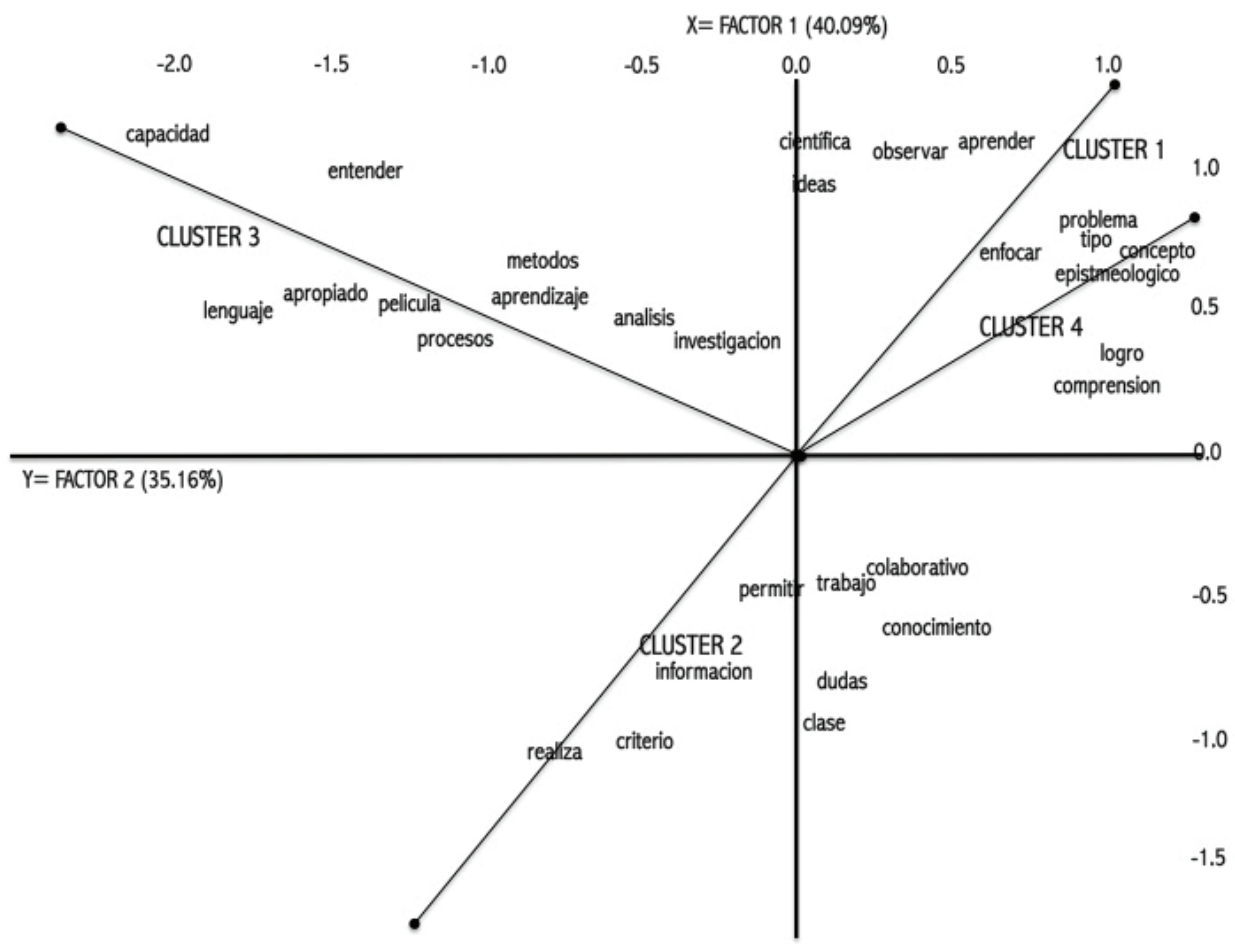

Figura 1. Biplot que representa los clúster formados a partir de la pregunta: De qué modo el cine favoreció la comprensión de los saberes epistemológicos vinculados con la investigación 
Cuadro 2. Descripción de los clúster derivados de la pregunta 1

\begin{tabular}{|c|c|c|c|c|c|c|}
\hline Clúster & Lemas & $\mathrm{Chi}^{2}$ & $\mathrm{P}$ valor & Lemas & $\mathrm{Chi}^{2}$ & $\mathrm{P}$ valor \\
\hline \multirow[t]{2}{*}{1} & Científica & 12.188 & 0.000 & Aprender & 21.500 & 0.000 \\
\hline & Ideas & 6.697 & 0.010 & Observar & 17.157 & 0.000 \\
\hline $\begin{array}{l}\text { Lemas en } \\
\text { contextos } \\
\text { según su } \\
\text { valor de } \\
\text { importancia } \\
\text { o Score. }\end{array}$ & \multicolumn{6}{|c|}{$\begin{array}{l}\text { Se aprenden conceptos mediante la observación de acciones. } \\
\text { Aprendimos los procesos de investigación científica como: identificación de un pro- } \\
\text { blema, el contexto del problema, variables, resultados. } \\
\text { Se observa cómo se hace investigación científica partiendo de hechos reales. } \\
\text { Se aprende a investigar con menor esfuerzo y más rendimiento. } \\
\text { Con el vídeo se observan los problemas desde diferentes puntos de vista. } \\
\text { Se fomenta el cruce de ideas. El trabajo con vídeos favorece el empoderamiento de } \\
\text { conceptos epistemológicos. }\end{array}$} \\
\hline Clúster & Lemas & $\mathrm{Chi}^{2}$ & $\mathrm{P}$ valor & Lemas & $\mathrm{Chi}^{2}$ & $\mathrm{P}$ valor \\
\hline \multirow[t]{4}{*}{2} & Dudas & 8.469 & 0.004 & Clase & 8.377 & 0.004 \\
\hline & Criterio & 7.040 & 0.008 & Conocimiento & 6.468 & 0.011 \\
\hline & Realizar & 5.618 & 0.018 & Información & 4.311 & 0.038 \\
\hline & Permitir & 3.949 & 0.047 & & & \\
\hline $\begin{array}{l}\text { Lemas en } \\
\text { contextos } \\
\text { según su } \\
\text { valor de } \\
\text { importancia }\end{array}$ & \multicolumn{6}{|c|}{$\begin{array}{l}\text { El análisis y síntesis se realizan de forma más efectiva, mejorando la calidad de los } \\
\text { trabajos finales. } \\
\text { La interacción permite aclarar dudas, fortalecer limitaciones conceptuales y muestra } \\
\text { diferentes formas de enfrentar el proceso de investigación. } \\
\text { Comparten diversidad de criterios para asumir aquellos que más se acerquen a nues- } \\
\text { tra posición sobre el conocimiento. } \\
\text { Se produce un acercamiento colaborativo al conocimiento de la investigación. } \\
\text { Enseña que la investigación se puede desarollar desde cualquier punto o conoci- } \\
\text { miento previo. } \\
\text { Se hacen abstracciones a partir de la realidad. }\end{array}$} \\
\hline Clúster & Lemas & $\mathrm{Chi}^{2}$ & $\mathrm{P}$ valor & Lemas & $\mathrm{Chi}^{2}$ & $\mathrm{P}$ valor \\
\hline \multirow[t]{5}{*}{3} & Capacidad & 25.106 & 0.000 & Entender & 18.547 & 0.000 \\
\hline & Proceso & 15.863 & 0.000 & Aprendizaje & 13.527 & 0.000 \\
\hline & Apropiado & 12.648 & 0.000 & Lenguaje & 12.648 & 0.000 \\
\hline & Película & 9.026 & 0.003 & Investigación & 5.611 & 0.018 \\
\hline & Métodos & 4.419 & 0.036 & & & \\
\hline $\begin{array}{l}\text { Lemas en } \\
\text { contextos } \\
\text { según su } \\
\text { valor de } \\
\text { importancia. }\end{array}$ & \multicolumn{6}{|c|}{$\begin{array}{l}\text { Libertad de investigación lo que significa que las metodologías son cambiantes y } \\
\text { que se deben adaptar al objeto de la investigación y sus características. } \\
\text { Trabajar con la película permitió aprender diferentes métodos de investigación como } \\
\text { el deductivo, empírico, cualititativo. } \\
\text { Entender que la investigación no debe ahogarse con ideas prestablecidas }\end{array}$} \\
\hline
\end{tabular}




\begin{tabular}{|l|l|c|c|c|c|c|}
\hline Clúster & Lemas & Chi $^{2}$ & P valor & Lemas & Chi $^{2}$ & P valor \\
\hline 4 & Epistemológicos & 31.138 & 0.000 & Enfocar & 16.388 & 0.000 \\
\cline { 2 - 7 } & Concepto & 16.220 & 0.000 & Comprensión & 8.042 & 0.005 \\
\cline { 2 - 6 } & Logro & 5.299 & 0.021 & Problema & 3.840 & 0.050 \\
\hline $\begin{array}{l}\text { Lemas en } \\
\text { contextos } \\
\text { según su } \\
\text { valor de } \\
\text { importancia. }\end{array}$ & $\begin{array}{l}\text { El desmontaje del vídeo permite comprender los conceptos y tipos de enfoques } \\
\text { epistemológicos en el estudio de un mismo objeto. } \\
\text { Se identifican conceptos claros y precisos vinculados a los enfoques epistemológicos. } \\
\text { Se fortalece el conocimiento de los enfoques epistmológicos y su vinculación con el } \\
\text { conocimiento científico. } \\
\text { Se logró apreciar los enfoques epistemológicos y sus características desde un plano } \\
\text { práctico. } \\
\text { Se asimilan los conceptos epistemológicos asociados a la investigación. } \\
\text { Se comprende la flexibilidad y dominio de los enfoques epistemlógicos en la } \\
\text { investigación científica. }\end{array}$ \\
\hline
\end{tabular}

Pregunta 2: De qué modo el uso del cine fomentó cambios conceptuales respecto al aprendizaje de saberes epistemológicos y su vinculación con la investigación.

La figura 2 evidencia la formación de dos factores que explican el 59.02\% de la varianza asociada al discurso de los docentes que describieron el beneficio del cine como promotor de cambios conceptuales respecto al conocimiento de la epistemología y su vinculación con la investigación. Alrededor de estos factores se construyen 5 clúster (ver cuadro 3) que a continuación se significan, como se mencionó en el apartado de la pregunta 1, mediante la vinculación del lema en su contexto discursivo:

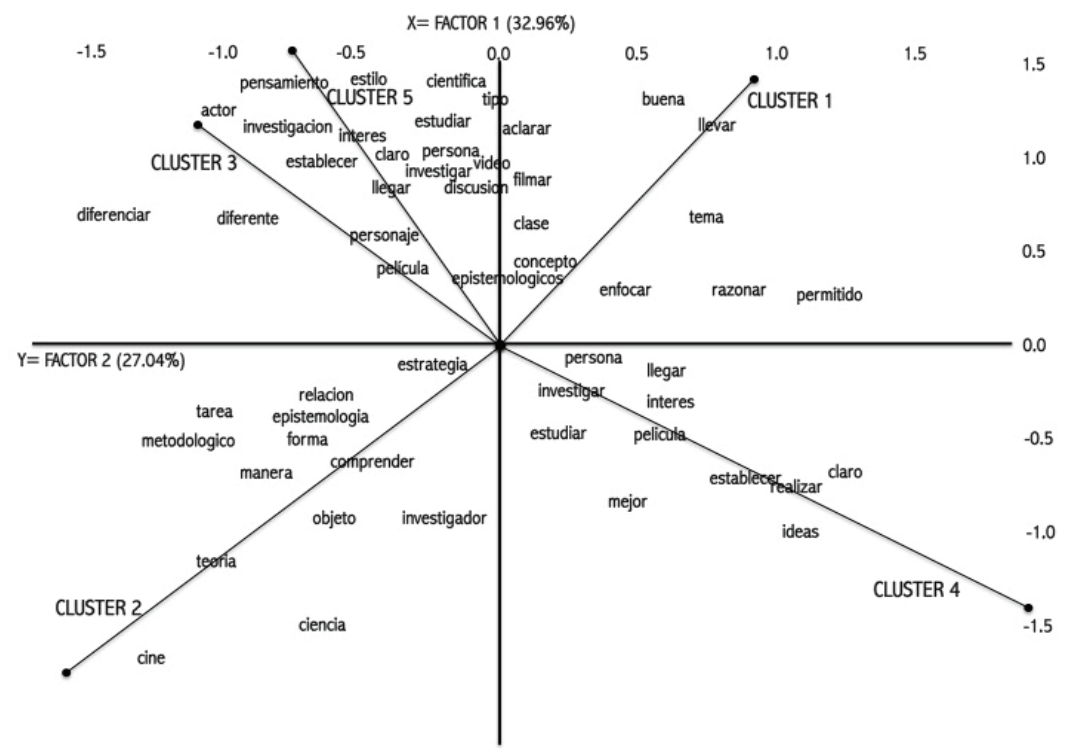

Figura 2. Biplot que representa los clúster formados a partir de la pregunta: De qué modo el cine fomentó cambios conceptuales respecto al aprendizaje de saberes epistemológicos y su vinculación con la investigación. 
El clúster 1 revela la comprensión de la vinculación entre los enfoques epistemológicos y los procesos de investigación, lo cual ratifica el reconocimiento de que la investigación y sus procesos se encuentran en correspondencia con la epistemología. El segundo clúster hace hincapié en la existencia de vínculos diversos entre el sujeto y el objeto de la investigación, sólo que en esta oportunidad, la presencia del verbo "comprender" sugiere el indicio del cambio, como se valora en el trabajo de Quirantes Sierra, (2011). El docente comprende la multiplicidad de vínculos que se forman entre sujeto y objeto, lo cual está condicionado y legitimado por los enfoques epistemológicos. El tercer clúster, acentúa la noción de diversidad en los modos de hacer investigación. El cuarto clúster, en esta misma línea semántica, develan los modos diferenciados de operar y condicionar las decisiones que se toman en investigación en correspondencia con la perspectiva epistemológica de referencia. Hilado a estas ideas, el clúster 5 muestra que cada enfoque epistemológico tiene estilos de pensamiento asociados a los procesos metodológicos de cuya interacción se explican los mecanismos para llegar al origen de la verdad.

Cuadro 3. Descripción de los clúster derivados de la pregunta 2

\begin{tabular}{|c|c|c|c|c|c|c|}
\hline Clúster & Lemas & $\mathrm{Chi}^{2}$ & $P$ valor & Lemas & $\mathrm{Chi}^{2}$ & P valor \\
\hline \multirow[t]{5}{*}{1} & Buena & 27.387 & 0.000 & Tema & 17.895 & 0.000 \\
\hline & Tipo & 7.496 & 0.006 & Clase & 6.164 & 0.013 \\
\hline & Vídeo & 5.553 & 0.018 & Concepto & 4.998 & 0.026 \\
\hline & Aclarar & 4.938 & 0.026 & Filmar & 4.938 & 0.026 \\
\hline & Llevar & 4.983 & 0.026 & Permitir & 4.983 & 0.026 \\
\hline $\begin{array}{l}\text { Lemas en } \\
\text { contextos } \\
\text { según su } \\
\text { valor de } \\
\text { importancia }\end{array}$ & \multicolumn{6}{|c|}{$\begin{array}{l}\text { Se reforzaron los conceptos sobre epistemología, enfoque y tipos de enfoques. Al } \\
\text { relacionarlos con la película se aclaron mis dudas sobre la vinculación entre ellos. } \\
\text { Comprendí la vinculación entre el conocimiento epistemológico y la investigación. } \\
\text { El trabajo con vídeos ha permitido que la necesidad obliga a razonar y eso es clave } \\
\text { para la investigación. }\end{array}$} \\
\hline Clúster & Lemas & $\mathrm{Chi}^{2}$ & P valor & Lemas & $\mathrm{Chi}^{2}$ & $\mathbf{P}$ valor \\
\hline \multirow[t]{5}{*}{2} & Cine & 34.319 & 0.000 & Ciencia & 33.648 & 0.000 \\
\hline & Teoría & 7.748 & 0.005 & Comprender & 9.858 & 0.002 \\
\hline & Objeto & 8.005 & 0.005 & Forma & 7.783 & 0.005 \\
\hline & Epistemología & 4.308 & 0.038 & Investigador & 3.869 & 0.049 \\
\hline & Tarea & 3.969 & 0.049 & & & \\
\hline $\begin{array}{l}\text { Lemas en } \\
\text { contextos } \\
\text { según su } \\
\text { valor de } \\
\text { importancia }\end{array}$ & \multicolumn{6}{|c|}{$\begin{array}{l}\text { La mezcla entre lo audiovisual y la teoría permitieron ver la aplicación de la } \\
\text { epistemología de una forma simple y didáctica. } \\
\text { La relación entre epistemología y los procesos metodológicos a través del cine } \\
\text { hicieron que la tarea fuera más comprensible,. } \\
\text { La epistemologia en la investigación permite identificar la forma de relación entre } \\
\text { sujeto y objeto de diferentes maneras. }\end{array}$} \\
\hline
\end{tabular}




\begin{tabular}{|c|c|c|c|c|c|c|}
\hline Clúster & emas & $\mathrm{Chi}^{2}$ & $P$ valor & Lemas & Chi $^{2}$ & $P$ valor \\
\hline \multirow[t]{3}{*}{3} & iferente & 22.358 & 0.000 & Personaje & 12.798 & 0.000 \\
\hline & Actor & 8.047 & 0.005 & Diferenciar & 8.047 & 0.000 \\
\hline & Investigación & 4.682 & 0.030 & Película & 4.352 & 0.037 \\
\hline $\begin{array}{l}\text { Lemas en } \\
\text { contextos } \\
\text { según su } \\
\text { valor de } \\
\text { importancia }\end{array}$ & \multicolumn{6}{|c|}{$\begin{array}{l}\text { Trabajar con vídeos fue fundamental para diferenciar las bases epistemológicas a } \\
\text { partir de ejemplos reales de la investigación visualizados en los personajes de la } \\
\text { película. } \\
\text { Hay múltiples maneras de hacer investigación, lo cual se ve en el trabajo de los } \\
\text { actores de la película. } \\
\text { Se perciben diversas maneras de hacer investigación según los enfoques } \\
\text { epistemológicos. } \\
\text { Los personajes y sus roles permitieron entender el proceso de investigación y sus } \\
\text { productos, así como las técnicas e instrumentos que utilizamos. }\end{array}$} \\
\hline Clúster & Lemas & $\mathrm{Chi}^{2}$ & $\mathrm{P}$ valor & Lemas & $\mathrm{Chi}^{2}$ & $\mathrm{P}$ valor \\
\hline \multirow[t]{3}{*}{4} & Claro & 21.135 & 0.000 & Establecer & 21.335 & 0.000 \\
\hline & Ideas & 10.540 & 0.001 & Mejor & 5.283 & 0.022 \\
\hline & Realizar & 4.428 & 0.035 & & & \\
\hline $\begin{array}{l}\text { Lemas en } \\
\text { contextos } \\
\text { según su } \\
\text { valor de } \\
\text { importancia }\end{array}$ & \multicolumn{6}{|c|}{$\begin{array}{l}\text { A través de las escenas se pudo establecer los escenarios asociados al modo de } \\
\text { operar de los enfoques epistemológicos. } \\
\text { Los enfoques ofrecen claridad en las decisiones que se toman al momento de } \\
\text { investigar. } \\
\text { El vídeo ofrece un claro entendimiento de las ideas epistemológicas. }\end{array}$} \\
\hline Clúster & Lemas & $\mathrm{Chi}^{2}$ & $\mathrm{P}$ valor & Lemas & $\mathrm{Chi}^{2}$ & $\mathrm{P}$ valor \\
\hline \multirow[t]{6}{*}{5} & Investiga & 21.758 & 0.000 & Científica & 13.471 & 0.000 \\
\hline & Estudiar & 12.442 & 0.000 & Pensamiento & 12.442 & 0.000 \\
\hline & Estilo & 9.366 & 0.002 & Llegar & 9.366 & 0.002 \\
\hline & Interés & 9.241 & 0.002 & Inve & 6.518 & 0.011 \\
\hline & Conocer & 6.144 & 0.013 & Epistemológico & 6.144 & 0.013 \\
\hline & Persona & 4.656 & 0.031 & Película & 4.056 & 0.031 \\
\hline $\begin{array}{l}\text { Lemas en } \\
\text { contextos } \\
\text { según su } \\
\text { valor de } \\
\text { importancia }\end{array}$ & \multicolumn{6}{|c|}{$\begin{array}{l}\text { La película favoreció la familiarización de conceptos y categorías inmersos en la } \\
\text { investigación científica y por ende favoreció el conocer las corrientes epistemológicas } \\
\text { asociadas a la investigación. } \\
\text { La utilización de la película me enfocó en diversos estilos de pensamientos que } \\
\text { tienen presencia cuando investigamos. } \\
\text { El trabajo con vídeos permitió conocer los diversos estilos de pensamientos de } \\
\text { acuerdo con el enfoque espistmeológico de referencia para la persona. } \\
\text { Conocí que en cada enfoque epistemológico existen técnicas diversas para llegar a } \\
\text { conocer el origen de la verdad. }\end{array}$} \\
\hline
\end{tabular}


De acuerdo con lo expuesto, el uso del cine como vehículo de enseñanza de la reflexión epistemológica le concede al novel docente-investigador la posibilidad de reconocer múltiples caminos para llegar a la investigación; incluso, le ofrece la posibilidad de observar modos de singularizarla con su manera de pensar y de construir la naturaleza de su objeto de estudio. Aprender a través del cine promueve cambios de actitud cuando se emplea con fines pedagógicos (Petit y Solbes, 2012).

Los docentes estudiados lograron resignificar el uso de los conceptos epistemológicos a fin de liberar el método de los modos dogmáticos con los que se aplican en sus nichos académicos; evidencia de ello fue que encontraron medios para ofertar la diversidad de caminos para hacer conocimiento. Ratificaron que no existen "únicas vías" para realizar investigación; contrario a ello, avizoraron que concurren tantos caminos como construcciones cognitivas sean posibles de viabilizar (Feyerabend, 1987). Aprender a través del cine generó condiciones para entender que en lo diverso de una realidad hay objetos de estudio, por tanto, hay que voltear a mirar lo diverso que somos, nuestras riquezas multiculturales, cuando de formar investigadores y de hacer investigación se trata (Garritz, 2010).

En el discurso de los docentes se aprecia la comprensión de que sus epistemologías personales se hacen presentes al momento de investigar. Aprender epistemología con el cine, ratificó la presencia del propio enfoque epistémico, por tanto, se devela que lo epistémico es plural y a pesar de las fuertes tendencias empíricas y deductivistas observadas (Martínez Galaz y González Weil, 2014) hubo acuerdos en aceptar que toda práctica de investigación y de aprendizaje es única y, por tanto, está permeada de los rasgos de su autor, tal como lo sugieren los resultados de Benarroch y Marín, (2011). Poder visualizar en las películas trabajadas, cómo se van formando las ideas, promovió conflictos representados en los actores, haciendo que sus soluciones sean caminos alternativos para construir argumentos fiables y válidos en medio del desarrollo de la investigación. El cine como aliado de la enseñanza resignifica sus funciones. Como sugiere Carretero, (2011) sirve para ilustrar las visiones que se quieren transmitir de la ciencia y del trabajo científico, abriendo interesantes debates que rompen la pasividad del estudiante al vincular las herramientas de análisis de la realidad con la realidad ilustrada a través del film objeto de análisis (García Borrás, 2011).

A modo de conclusión, es importante destacar que la viabilidad de uso del cine como escenario para enseñar la reflexión metacientífica o epistemológica se soporta en su discursividad y de allí la diversidad de affordances o usos que hemos descrito. Este argumento se centra en el supuesto de que los conceptos epistemológicos se traman como discursos y terminan circulando en medio de las comunidades científicas. Por lo tanto, presentan condiciones y espacios de producción de significados, relaciones entre objetos, contrastes contextuales (Burgos y Nidia, 1993). El saber epistémico está inmerso en una totalidad de significados. Cada investigador desarrolla su propio sentido de decodificación para traducir las abstracciones percibidas en representaciones cognitivas. Como sugiere Van Dijk, (2005) la entrada lingüística se transforma en información semántica tan pronto como se contacta con la dimensión cognitiva del sujeto del discurso.

Justamente en este interfaz, tiene sentido abordar el uso del cine como medio de representación del modo en que opera la epistemología. El conjunto de señales visuales y auditivas alertan los sentidos y las ideas empiezan a resignificarse. En la medida en que se personalizan los conceptos, los anclajes cognitivos dan paso al insight y al 
igual que a Arquímedes con su "eureka", empiezan a vincularse piezas semánticas, que aisladas no transmitían nada. En este sentido, los affordance del cine como medio de representación meta-científica permiten una mayor comprensión de conceptos epistemológicos y metodológicos, involucran al formando con la autocorrección de dudas desde la cotidianidad de su percepción, impulsando la construcción de conceptualizaciones significantes; personaliza las abstracciones que subyacen al pensamiento epistemológico, se promueve la construcción de dispositivos lingüísticos que sirven de argumentos en las decisiones vinculadas con el desarrollo de la investigación.

El novel docente-investigador, mediante el uso del cine, tiene la oportunidad de hacer una disección al interior de la dinámica socio-epistemológica de los procesos científicos y así tener nociones del modo en que funcionan, para tomar decisiones congruentes con su esencia y su epistemología cuando de aprender, enseñar y vivir la investigación se trate.

\section{REFERENCIAS BIBLIOGRÁFICAS}

Álvarez Ortega, F. (1999). El problema de la verdad: una aproximación analítica. México: Universidad Iberoamericana.

Acevedo-Díaz, J., Vazquez-Alonso, A., Manassero-Mas, M., y Acevedo-Romero, P. (2007). Consensos sobre la naturaleza de la ciencia: Aspectos Epistemológicos. Revista Eureka. Enseñanza y divulgación de la ciencia, 4 (2), 202-225.

Altisen, C. (2001). Epistemología. La Plata: UCRLP.

Apted, M. (Director) y Clegg, T., Glimcher, A. (Producers). (1988). Gorilas en la niebla: la historia de Dian Fossey [Imagen en movimiento]. EEUU.

Bacon, F. (2011). La gran restauración (Novum Organum). (M. A. Granada, Trans.) Madrid, España: Tecnos.

Bacon, F. (2006). Nueva Atlantida. (E. García Estébanez, Ed.) Madrid, España: Akal.

Baerentsen, K., \& Trettvik, J. (October de 2002). An activity theory approach to affordance. NordiCHI , 19-23.

Barello, S., Leone, D., Danese, S., \& Vegni, E. (2014). Inflammatory bowel diseases and psicological issues: A new approach for a systematic analysis of te academic debate. Psycology, Health \& Medicine, 19 (5), 559-571.

Benarroch, A., y Marín , N. (2011). Relaciones entre las creencias sobre enseñanza, aprendizaje y conocimiento de las ciencias. Enseñanza de las ciencias, 29 (2), 289-304.

Biber, H., \& Nagy, S. (2010). Mixed methods Research. New York, EEUU: The Guilford Press.

Borghi, A., \& Riggio, L. (2009). Sentence comprehension and simulation of object temporary, canonical and stable affordances. Brain Research , 117-128.

Bunge, M. (2002). Epistemología. Barcelona: Siglo XXI.

Burgos, B., y Nidia, R. (1993). Análisis del discurso y educación. Documentos DIE, 26.

Camacho, H. (2000). Enfoques epistemológicos y secuencias operativas de investigación. Maracaibo, Venezuela: Universidad Rafarel Belloso Chacín.

Caputo, A. (2013). Cultural models shaping stalking from a content analysis of italian newspapers. Europe`s Journal of psicology, 9 (3).

Carretero, M. (2011). Cine y literatura como herramientas de trabajo para el estudio de enfermedades contagiosas. Revista Eureka sobre enseñanza y divulgación de las ciencias, 8 (3), 341-352.

Carrillo Guerrero, L. (2006). La (lógica) construcción de la realidad. Ámbitos (15), 12-156.

Castro Nogueira, L., Castro Nogueira, M., \& Morales Navarro, J. (2008). Metodología de las ciencias sociales. Una introducción crítica (2da ed.). España: Tecnos.

Chá Larrieu, A. (2002). Elementos de Epistemología. Uruguay: Ediciones TRILCE. 
Costall, A. (1995). Socializing affordances. Theory \& psycology, 5 (4), 467-481.

Da silva Oliveira, F., y Tosi Rodigues, S. (2006). Affordances: a relação entre ambiente e agente. Ciencias y cogniçao , 9, 120-130.

De Vries, J. (1945). Pensar y Ser. Madrid: Razón y Fe.

Dear, P. (2001). La revolución de las ciencias. El conocimiento europeo y sus expectativas, 15001700. (J. Marcaida López, Trans.) Madrid, España: Marcial Pons, Ediciones de Historia, S.A.

Descartes, R. (2009). Discurso del método. (M. Caimi, Trans.) Buenos Aires, Argentina: Colihue.

Echeverría , J. (1998). Filosofía de la ciencia. España: Akal.

Estany, A. (2006). Introducción a la filosofía de la ciencia. España: Universitat Autónoma de Barcelona.

Fernández, I., Gil, D., Carracosa, J., Cachapuz, A., \& Praia, J. (2002). Visiones deformadas de la ciencia transmitidas por la enseñanza. Historia y epistemología de las ciencias, 20 (3), 477-488.

Feyerabend, P. (2008). Adiós a la razón. España: Tecnos.

Feyerabend, P. (1987). Contra el método. España: Ariel.

García Borrás, F. (2011). Las escenas cinematográficas: una herramienta para el estudio de las concepciones alternativas de física y química. Revista Eureka sobre enseñanza y divulgación de las ciencias, 8 (3), 291-311.

Garritz, A. (2010). Enseñanza de la ciencia en una sociedad con incertidumbre y cambios acelerados. Enseñanza de las ciencias, 28 (3), 315-326.

Guerrero, G. (2009). Introducción a la filosofía de la ciencia. Santiago de Cali: Universidad del Valle. Gibson, E. (2000). Where Is the Information for Affordances? Ecological Psycology, 12 (1), 53-56.

Hume, D. (1984). Tratado de la naturaleza humana (Vol. 18). Barcelona: Orbis.

Hutchby, I. (2001). Technologies, texts and affordances. Sociology, 35 (2), 441-456.

Izquierdo, A. (2000). Friederich Nietzcche. Madrid, España: EDAF.

Kuhn, T. (2004). Las estructura de las revoluciones científicas. México: FCE.

Kuhn, T. (1989). ¿Qué son las revoluciones científicas? Y otros ensayos. (J. Romo Feito, Trans.) España: Paidos.

Lakatos, I. (1999). The methodology of scientific research programmes. (J. Worral, \& G. Currie, Eds.) UK: Cambridge University Press.

Lakatos, I. (1976). Proofs and Refoutations. The logic of mathematical discovery. UK: Cambridge University Press.

Laudan, L. (1978). Progress and its problems. Towards a theory of scientific Growth. EEUU: University of California Press.

Locke, J. (1985). Ensayo sobre el entendimiento humano (Vol. 96). Barcelona: Orbis.

Losey, J. (Director). (1972). Galileo [Motion Picture]. Reino Unido.

Marín, A., y Branch, J. (2008). Aplicación de dos nuevos algoritmos para agrupar resultados de búsquedas en sistemas de catálogos públicos en línea (OPAC). Revista interamericana de bibliotecología, 31 (1), 47-65.

Martínez Galaz, C., y González Weil, C. (2014). Concepciones del profesorado universitario acerca de la ciencia y su aprendizaje y cómo abordan la promoción de competencias científicas en la formación de futuros profesores de Biología. Enseñanza de la ciencia, 32 (1), 51-81.

Miller, G., Enright, N. (Productor), y Miller, G. (Director). (1992). El aceite de la vida [Imagen en movimiento].

Nicolás, J. A., y Frápoli, M. J. (1997). Teorías de la verdad en el siglo XX. Madrid: Tecnos.

Nietzsche, F. (2000). La voluntad de poder. (M. Prieto, Ed., \& A. Froufe, Trans.) España: Edaf.

Nussbaum, M. (2003). La terapia del deseo (Vol. 119). Barcelona: Paidós.

Olmedo, F. (1992). Los presocráticos (Vol. 1). Quito: Libresa.

Orden Jiménez, R. (1998). El sistema de la filosofía de Krause. Génesis y desarrollo del panteísmo. España: Aldecoas.

Padrón, J. (2007). Tendencias epistemológicas de la investigación científica en el siglo XXI. Cinta 
moebio, 28, 1-32. http://www.moebio.uchile.cl/28/padron.html

Paz Penagos, H. (2008). Visiones deformadas de la ciencia y la enseñanza-aprendizaje de conceptos científicos. Crítica a prólogos de textos-guía de comunicaciones electrónicas digitales. REVISTA Universidad EAFIT, 44 (149), 23-37.

Petit, M., y Solbes, J. (2012). La ciencia ficción y la enseñanza de las ciencias. Enseñanza de las ciencias: revista de investigación y experiencias didácticas, 30 (2), 55-72.

Platón (1992). Diálogos escogidos (Vol. 2). Quito: Libresa.

Quesada Sánchez, F. J. (2004). Aproximación a la metodología de la ciencia: Las ciencias sociales y la contabilidad. España: Universidad Castilla La Mancha.

Quirantes Sierra, A. (2011). Física de Película: una herramienta docente para la enseñanza de Física universitaria usando fragmentos de películas. Revista Eureka sobre enseñanza y divulgación de las ciencias, 8 (3), 334-340.

Ramírez Sánchez, S. L. (2002). La idea de tradición en Kuhn. En: A. Velasco Gómez, Perspectivas y horizontes de la filosofía de la ciencia a la vuelta del tercer milenio. México: Universidad Nacional Autónoma de México.

Torres-Moreno, J.-M., Molina, A., y Sierra, G. (2010). La energía textual como medida de distancia en agrupamiento de definiciones. JADT: 10th International Conference on Statistical Anallysis of Textual Data, 215-226.

Torruella, J., y Llisterri, J. (1999). Diseño de corpus textuales y orales. In J. M. Blecua, G. Clavería, C. Sanchez, \& J. Torruella, Filología e informática. Nuevas tecnologías en los estudios filológicos (pp. 45-77). España: Universidad Autónoma de Barcelona.

Turvey, M. T. (1992). Affordances and Prospective Control: An Outline of the Ontology. Ecological Psychology, 4 (3), 173-187.

Van Dijk, T. (2005). Estructuras y funciones del discurso. México: Siglo XXI Editores. 